arguments are based upon experience at ophthalmic hospitals. In a well-equipped general hospital, where a patient, referred by the ophthalmic surgeon, is admitted to a medical ward, and subjected to examination, often painfully thorough, by the physician and by the staff of the clinical, chemical, and bacteriological laboratories, there would be no scope for the energies of an ophthalmic physician.

\title{
Blindness due to Ophthalmia Neonatorum
}

The News Letter, the official organ of the National Committee for the Prevention of Blindness (New York) has recently (October, 1919) published the statistics of 1918-19 dealing with the admissions to the State Schools for the Blind, and with schools and day classes for the blind in various cities in the United States. The figures are interesting, since those for ophthalmia neonatorum are distinguished from the others. As regards 34 State Schools the total admissions for the two years was 3,537 children, of whom 89 (or 15.7 per cent.) had been blinded by ophthalmia neonatorum. The highest percentage of admissions from ophthalmia was in the Mississippi Institution for the Blind, where it reached the astonishing figure of 85.7 per cent., but then the significance of that figure is much diminished when we find that the total admissions were seven only, of which six were due to the disease in question. Eleven of the institutions had no ophthalmia admissions. Of 56 admissions into 10 schools and classes for the blind in the various cities 16 (or 28.6 per cent.) were admitted on account of the results of ophthalmia. From another table given by our contemporary, showing the number of pupils newly admitted in Schools for the Blind during the past eleven years who were blind from ophthalmia neonatorum, it appears that admissions from ophthalmia ranged from 26.6 per cent. of the admissions in $1907-8$ to 14.7 per cent. in 1917-18. The percentage may, broadly speaking, be said to have fallen year by year, a most satisfactory result.

\section{Colour-Matching by "Artificial Daylight"}

The correct matching of colours is a problem of great interest, both from the physical and physiological sides, and has been appropriately the subject of study by the Illuminating Engineering Society on several occasions. Assuming that the operator engaged in matching colours has normal colour vision, he in general requires for his work a "white" light, approaching that of the difiused north sky. The ideal natural lighting conditions, however, are 